\title{
A Model for Estimating the Anisotropy of the Conduction Velocity in Cardiac Tissue Based on the Tissue Morphology
}

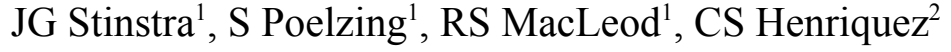 \\ ${ }^{1}$ University of Utah, Salt Lake City, UT, USA \\ ${ }^{2}$ Duke University, Durham, NC, USA
}

\begin{abstract}
This paper describes a modeling technique for studying propagation in cardiac tissue at a microscopic level. The model consisted of realistically shaped myocytes embedded in an extracellular matrix and coupled by means of gap junctions. The model was used to estimate propagation velocities along and across the fiber structure of the myocardium, specifically to estimate the effects of altering the amount of extracellular space that separates the myocytes. The model shows that shrinking the extracellular space causes propagation to slow down along the fiber, but to speed up across the fiber structure of cardiac tissue.
\end{abstract}

\section{Introduction}

In the quest to better understand the mechanisms underlying cardiac arrhythmias, the use of computer simulations is a common tool. Computer simulations have the advantage of being able to resolve potentials and currents with a finer level of detail than is possible with measurements. However, in order to be useful, simulations need to reproduce observations, in this case from electrophysiology, and hence they must produce results against which to compare with experimental findings.

The most common approach to the simulation of the propagation of depolarization fronts inside the myocardium is the use of the "bidomain model" [1]. The bidomain model is based on the notion that the myocardium can be separated into intracellular and extracellular spaces, which are joined to each other by a membrane that acts both as a current source and a pathway allowing current to flow between both spaces. More specifically, the bidomain model assumes that the intracellular space, the extracellular space and the membrane all coexist at each point in space. Hence at each location the myocardium can be characterized by a conductivity tensor for the intracellular space, a conductivity tensor for the extracellular space, and an ionic model that describes the current flowing through the membrane.
The two conductivity tensors describing the electrical properties of the tissue represent the homogenized conductivity of either the intracellular or the extracellular space. The most common way of estimating these parameters is to choose them so that simulated cardiac propagation speeds fit experimentally observed values. Although this is a valid way of setting these parameters, it fails to relate them to the underlying tissue structure and composition.

In this paper, we describe a model that simulates cardiac propagation based on a more detailed description of the microscopic tissue morphology than that provided by the standard bidomain. One of the reasons for including more details into the model is to be able to simulate pathologies like ischemia based on their physiological origins rather than by assigning parameters to fit experiments. For instance, during an episode of]ischemia the amount of extracellular space changes with time [2] altering the way a depolarization front propagates along the fiber [3].

In order to create such a model, we built on the models of Spach et al. [4], who created a 2D model of cardiac tissue that consisted of several hundred realistically shaped myocytes that were coupled by gap junctions. Their model was limited by the fact that it did not include an extracellular space. Two-dimensional models are intrinsically limited because the intracellular and extracellular current paths cannot cross each other, thereby limiting the amount of available extracellular and intracellular pathways.

To avoid these limitations of a 2D model, we created a full 3D model of cardiac tissue that included realistically shaped myocytes coupled by gap junctions and embedded in an extracellular matrix. We present here preliminary simulations aimed at exploring the differences in cardiac conduction along and across myocardial fibers that make up the myocardium. We pay special attention to conduction perpendicular to the myocardial fiber structure, which is not fully understood and thought to be influenced by factors ranging from gap junctions to capacitive coupling between myocytes [5]. Our hypothesis is that reduction of the interstitial space affects 


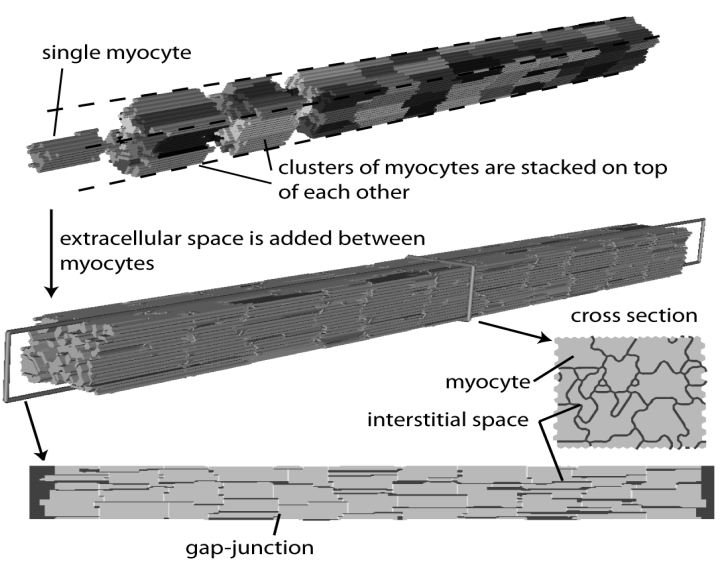

Figure 1 - Creating a computer generated geometry for the structure of cardiac tissue. The upper panel shows how geometrical shapes are grouped together to form layers of myocytes. The lower panel shows the final model with extracellular space carved out between the myocytes. The two lower right figures show a cross section through the computer generated model.

Model 1: simulation of wavefront along bundle

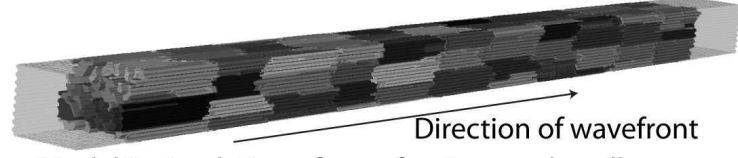

Model 2: simulation of wavefront across bundle

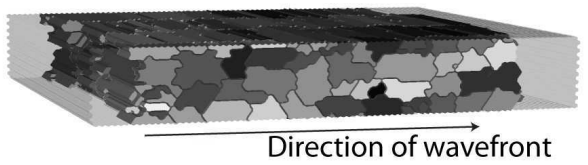

Figure 2 - Two separate geometrical models for simulating propagation along the fiber of the tissue and one for simulating propagation across the fiber. Note the models are displayed at a different scale.

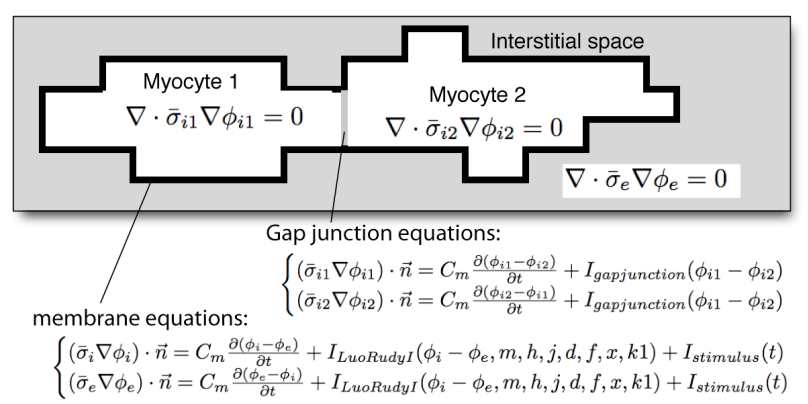

Figure 3 - Layout of the electrical model that is used in the simulations. The figure depicts the subdivision of the tissue into several small domains that each represent a volume conductor and which are coupled by means of their boundary conditions. propagation wavespeed differently along and across fibers.

\section{Methods}

Our model consisted of three components: (1) a geometrical model of cardiac tissue, (2) an electrical simulation process, and (3) a finite element solver that combined the geometrical and electrical model and computed potentials as a function of time.

The geometrical model that underlies the simulation was based on previous work that focused on computing passive electrical characteristics of myocardial tissue such as the conductivity tensor for a bidomain $[2,6]$. We created a computer algorithm for filling up a space with realistically shaped myocytes that fit together like pieces of a jigsaw puzzle of which the average length and cross section could be altered to fit histological data. The initial step of the algorithm filled the space completely with myocytes and in a second step space was carved out to generate an adjustable interstitial space between the myocytes. Figure 1 contains an example of such computer generated meshes. For the results presented here we generated two separate tissue geometries, one for conduction along the myocardial fiber and one for across the fiber (Figure 2). The reason for using two separate geometrical models was to constrain the number of cells needed in the model. In figure 2, an example of both models is given. For longitudinal propagation, we created a model with more cells along than across the fiber; stimulating the fiber at one end producs a stable depolarization front that spreads along the fiber after traversing a series of myocytes. Similarly, the second model was created that had more cells across the fiber bundle, in order to accurately simulate a wavefront spreading across the fiber.

For the example case shown in this paper we generated two different types of meshes with different amounts of extracellular space (an extracellular volume fraction of respectively $12 \%$ and $3 \%$ ). The latter could, for instance, reflect the collapse of the interstitial space during the onset of acute ischemia [2]. The extracellular space was carved out on the lateral sides of the myocytes (perpendicular to the fiber direction) and the amount of extracellular space was regulated by altering the thickness of the sheet of interstitial space that surrounds the myocytes. As no extracellular space was carved out at the long ends of the myocytes, these were touching the next layer of neighboring myocytes. It was assumed that where two myocytes shared a surface, this surface was infused with gap-junctions forming a conductive path between two myocytes.

The geometrical models were created from a lattice of hexagonal, hexahedral, and prism elements. The various geometrical elements that describe the tissue morphology were subsequently subdivided into tetrahedral elements, which are used as a basis of the computation of the potentials throughout the model. The resulting meshes consisted of about 300,000 nodes. 
Potential at membrane in model

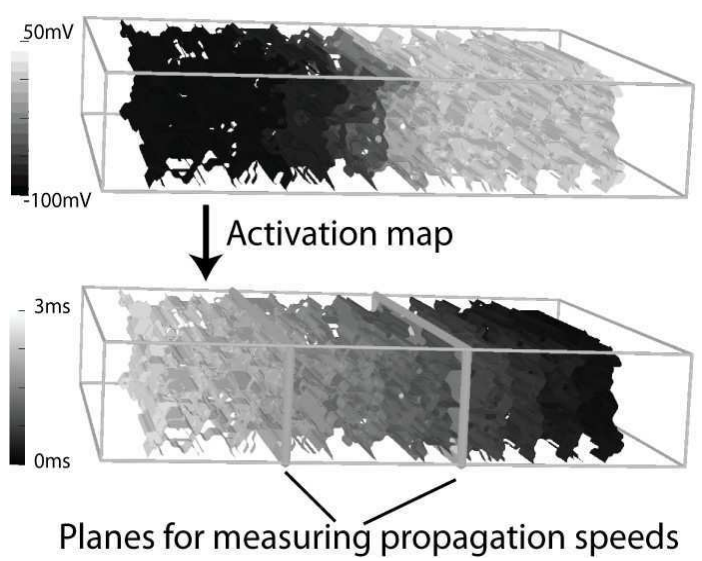

Figure 4 - Example of the simulations. The upper panel shows the transmembrane potential throughout the volume of the simulated piece of tissue. The lower panel shows the activation map and the two planes where we measured the average activation time for measuring propagation speeds.

For the electrical model, we assumed that each domain in the model could be described by an ohmic volume conductor, with a conductivity of $20 \mathrm{mS} / \mathrm{cm}$ for the extracellular space and a conductivity of $3 \mathrm{mS} / \mathrm{cm}$ for the intracellular space. We assumed that the membrane separating both spaces was infinitely thin and that the current flowing through the membrane could be approximated by a ionic membrane model that describes the current through the membrane as a function of the potential difference between both spaces and the internal state of the ion channels. This leads to a description in which the domains (each myocyte and the interstitial space) are a separate volume conductors that are connected to each other by means of shared boundary conditions. When the shared interface was between myocytes, we assumed a passive surface resistor, in case the shared interface was between the interstitial space and a myocyte we assume that the voltage current relation is described by the Luo-Rudy I membrane model that was modeled after ventricular myocyte of a guinea pig [7]. When the interface is an outer boundary a no-flux boundary condition is assumed. The model and its governing equations are summarized in figure 3 , which schematically depicts the subdivision in different spaces as well.

In order to solve the set of partial differential equations depicted in figure 3 , we used the finite element method. We used the geometrical mesh of the computer generated myocyte shapes as the basis for the computation. This mesh was sliced up into several domains by splitting the nodes at the interfaces between the various intracellular and extracellular domains. The split nodes were subsequently used as collocation points at which the membrane/gap-junction equations were evaluated based on the potential difference across the mesh. The latter equations served as common boundary conditions that linked the individual volume conductor models together.

The potentials in the model were solved using an semi-implicit time stepping scheme, in which current at the boundaries was solved first and was used to compute the potential distribution. The latter was used again to compute the currents that flow through the membrane. In order to reduce the number of time steps needed for computation we used an adaptive time stepping scheme with a linear estimator of the next step, in a scheme similar to the one published by Luo et al. [7]. We used an iterative solver for each time step using the MINRES algorithm.

The stimulation current was injected directly into the membrane and consisted of a $0.4 \mathrm{~ms}$ wide pulse that injected a homogeneously distributed current into the cell membranes. In order to stabilize the membrane equations we did run the simulation in a simple strand of myocytes with a similar intracellular to extracellular volume ratio, and used the average state of the membrane as an initializer for the model. In order to simulate propagation, we simulated the first $8 \mathrm{~ms}$ after stimulation.

Matlab (www.mathworks.com) was used to generate the geometries for the models, a modified version of CardioWave was used to run the simulation (cardiowave.duke.edu), and the SCIRun software was used to setup the finite element computation and to link all the components together (software.sci.utah.edu). Each simulation took about half a day to complete on a MacPro (two quad-core Xeon processors of each $3 \mathrm{GHz}$ ).

\section{Results}

To characterize the differences between conduction along the fiber structure of the tissue and across it, we simulated two different amounts of extracellular space: a volume fraction of $12 \%$, and one of $3 \%$ respectively. For each of these cases the tissue was stimulated at one end by a short pulse and the resulting wavefront inside the tissue piece was simulated. In order to measure the conduction velocity of the propagating wavefront we measured the time delay between the wavefront passing through two different surfaces located in the center of the fiber, as shown in figure 4 . We defined the wavefront as the location at which the average transmembrane potential in the cross section through the tissue is equal to a value of $-30 \mathrm{mV}$. By computing the time it took for the wavefront to pass between both surfaces and dividing the distance between both surfaces by this time delay, we estimated the conduction velocity. The resulting velocities for both fiber directions are summarized in table 1.

The results show: (1) that for the chosen configuration the conduction velocity across the cells increases and conduction velocity along the cells decreases when restricting the amount of extracellular space and 2) the conduction velocity along the cells is more sensitive to changes in extracellular space than the conduction 
velocity across the cells. The results indicate that the anisotropy ratio of the conduction velocity is a function of the amount of extracellular space in the tissue.

Table 1. Conduction velocities

\begin{tabular}{|c|c|c|}
\hline Conduction Velocity $(\mathrm{m} / \mathrm{s})$ & $\begin{array}{c}\text { Parallel to the } \\
\text { fiber }\end{array}$ & $\begin{array}{c}\text { Perpendicular } \\
\text { to the fiber }\end{array}$ \\
\hline large ECS $(12 \%)$ & 0.83 & 0.17 \\
\hline small ECS (3\%) & 0.51 & 0.19 \\
\hline
\end{tabular}

\section{Discussion and conclusions}

When the amount of extracellular space is reduced in the model, the sheets of extracellular space that laterally separate the the myocytes become thinner. The latter results in the resistance along these sheets in the direction of the fibre structure to be larger, which in turn leads to a larger extracellular voltage gradient along these sheets. In the case where we simulated propagation along the fiber structure of the tissue, the additional resistance along the extracellular sheets causes the potentials to fall off more rapidly along the fiber, and as well the added resistance causes the amounts of currents to be reduced in the extracellular space. This leads to a slower charging of the membranes in front of the depolarization front. The latter results in lower conduction velocities along the fiber. In the case of conduction across the fiber structure, the resistance across the thickness of the extracellular sheets becomes smaller, as the thin layer of extracellular space gets thinner. For the conduction across the fiber structure the currents flow both perpendicular to these extracellular sheets as well as along them. When the membrane of the myocyte on one side of the extracellular sheet depolarizes it absorbs currents from these small extracellular spaces, which leads to a drop in the potentials in this extracellular space. As the extracellular spaces are thin, they are poorly coupled to the rest of the extracellular matrix and the changes in the extracellular potentials will not only alter the transmembrane potentials on the side of where the cell gets depolarized, it will alter the the transmembrane potentials of the membrane on the other side as well. The latter leads to a coupling of cells over these small clefts, rendering a possible explanation of why cells get better coupled with a smaller amount of extracellular space.

In previous work, simulations aimed at computing the bulk conductivity of tissue using the same underlying tissue model showed that reducing the extracellular space raised the impedance both along as well as across the fiber [6]. Hence, the examples presented in this paper show that one cannot directly translate the changes in macroscopic tissue conductivity into the bidomain model, as the bidomain model predicts assumes a similar relation between resistance and propagation velocity for both directions.

Moreover the apparent tissue conductivity depends on the actual location of the current sources, e.g. for measuring macroscopic tissue conductivity the electrodes are far apart. However in the case of propagation the current sources are deeply embedded inside the tissue structure and hence the apparent impedances and current paths in this setting are different from the ones when measuring macroscopic impedances.

Experimentally a similar phenomena has been observed by Bursac et al. [8], who by means of microabrasion increased the amount of extracellular space and observed a speed up along the fiber structure and a slow down across the fiber structure.

In conclusion, the conduction velocity is affected by the distribution of the interstitial space and as such changes in the fraction of interstitial space due to disease of aging may have different effects on propagation along and across cells.

\section{Acknowledgments}

We want to acknowledge support for this work through NIH grants RO1 HL076767, P41- RR12553-07, and the Nora Eccles Treadwell Foundation.

\section{References}

[1] Henriquez CS. Simulating the electrical behavior of cardiac tissue using the bidomain model. Crit Rev Biomed Eng. 1993; 21(1): 1-77.

[2] Stinstra JG, Shome S, Hopenfeld B, MacLeod RS. Modelling passive cardiac conductivity during ischaemia. Med Biol Eng Comput. 2005; 43(6):776-82.

[3] Stinstra JG, Roberts SF, Pormann JB, MacLeod RS, Henriquez CS. A Model of 3D Propagation in Discrete Cardiac Tissue, Proc. Computers in Cardiology, 2006

[4] Spach MS, Barr RC. Effects of cardiac microstructure on propagating electrical waveforms, Circ Res. 2000: 86(2): E23-28

[5] Sperelakis N, An Electric Field Mechanism for Transmission of Excitation Between Myocardial Cells. 2002; Circ Res 91(11): 985-987

[6] Stinstra JG, Hopenfeld B, Macleod RS. On the passive cardiac conductivity. Ann Biomed Eng. 2005; 33(12):1743-51.

[7] Luo CH, Rudy Y. A model of the ventricular cardiac action potential. Depolarization, repolarization, and their interaction. Circ Res. 1991 Jun;68(6):1501-26.

[8] Bursac N, Parker NN, Iravanian S, Tung L, Cardiomyocyte cultures with controlled macroscopic anisotropy: A model for functional electrophysiological studies of cardiac muscle, Circ Res. 2002; 91: e45-e54

Address for correspondence:

Jeroen Stinstra

Scientific Computing \& Imaging Institute

University of Utah

50 S Central Campus Drive

Salt Lake City, UT 84112

USA

E-mail address (jeroen@sci.utah.edu) 